\title{
Nonlinear Dynamics of the Vehicle-Bridge Coupled Interaction System
}

\author{
C.Z. Qian, ${ }^{1,2}$ C.P. Chen, ${ }^{1,2, *}$ L. Hong ${ }^{1,2}$ and \\ G.W. Zhou ${ }^{1,2}$ \\ ${ }^{1}$ Shool of Civil Engineering and Architecture, Xiamen \\ University of Technology, Xiamen, Fujian Province, \\ China \\ ${ }^{2}$ Sino-Canada Research Center for Noise and Vibration \\ Control, Xiamen, Fujian, China
}

\begin{abstract}
Considering geometry nonlinear of the bridge, the differential equations of vehicle-bridge coupled interaction system are established. Using numerical method, the numerical solution of the coupling nonlinear differential equation are obtained. As an example, single vehicle pass through the bridge is studied. The result shows nonlinear items has little effects to the response in this case. In the other example, the train is modeled as serious spring-mass system pass through bridge. In this case, the subharmonic resonance are studied. The result shows when the bridge is excited period force, nonlinear affect can not be ignored.
\end{abstract}

Keywords. nonlinear dynamics, moving load, subharmonic resonance.

\section{Introduction}

The dynamics of the vehicle-bridge coupled interaction system has been one of the most interest researches for many years. The interest was at the beginning oriented toward bridges and railways to study the conditions under which these structures are stable [1,2]. It is proved in some studies [3-5], the dynamical displacement responses of the bridge under the moving vehicles are bigger than the static displacement under the static axle load. So, in the bridge design, especially in the railway bridge design, the impact effect of the train plays an important role. In order to ensure the safety and the serviceability of the bridge, it is inevitable to take the dynamic factors into account in predicting the deflection status of the bridge under the moving loads.

In recent decades, the research about vehicle-bridge interactions developed greatly. The researches are mainly focused on the problems as follows: (1) modeling the vehicles $[3,6,7]$, the vehicles are simplified as a constant moving

Corresponding author: C.P. Chen, E-mail: cpchen@126.com.

Received: 5 April 2013. Accepted: 4 June 2013.

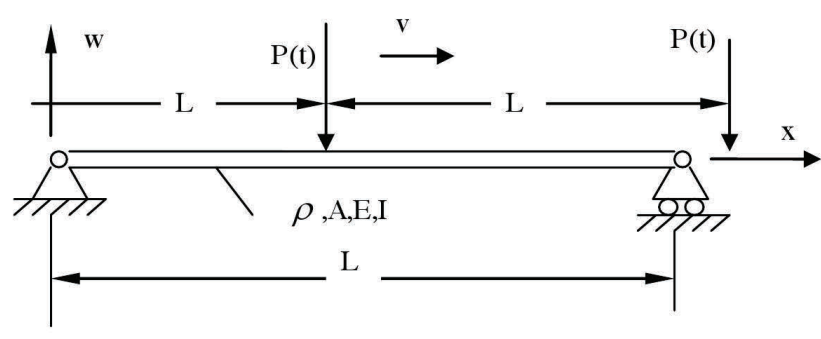

Figure 1. Bridge model with moving force.

force, a moving mass, a serious of axles or serious of moving spring-mass system. (2) modeling the bridges [7-11], studied bridges are considered as simple supported beam, continuous beam, suspension bridge and cable bridge. (3) vibration control for vehicle-bridge system [12-15].

Compared with above several aspect of research, few literature talk about the nonlinear dynamics of the vehiclebridge system. In fact, some experimental research appears that nonlinear effect play the important role as the amplitude of oscillations increases [16]. In nature, the source of nonlinearity is various, which may be inertial, geometric or material, the influence of the nonlinearity on the beam dynamics should also be highlighted. Nonlinear dynamical problems are so complex that few problem can be solved by the analytic method. Perturbation method is applied in single degree of freedom, multiple degree freedom, and some infinite degree of freedom system [17]. But for the complex nonlinear system, numerical solution is the main method.

In this paper, the dynamical problems of vehicle-bridge system are studied with considering geometry nonlinear of the bridge. The vehicles are modeled as series of constant force or series of spring-mass system. Numerical method is applied for solving the response data of the system. The results are contracted with the case not considering the nonlinear. The subharmonic resonance of the system are discussed and some useful conclusions are obtained.

\section{Formulation of theory}

The bridge model adopted is shown in Fig. 1. In discussing beams, attention is restricted to planar and non-rotating motions. The equations of motion are obtained by a combination of the inertial effect of the moving load and the nonlinear effect in the beam dynamics. Assuming that plane 


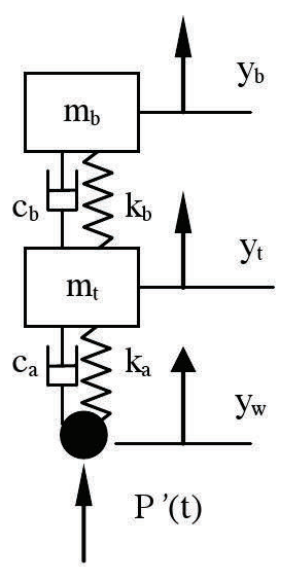

Figure 2. Vehicle model.

sections remain plane and a linear stress-strain law, the equations of motion governing the non-linear dynamics of a beam with uniform shape subjected to a moving load $P$ and velocity $v$ are given by

$$
\begin{aligned}
& \rho A u_{t t}-E A u_{x x}=\frac{1}{2}(E A) \frac{\partial}{\partial x}\left[\left(1-2 u_{x}\right) w_{x}^{2}\right] \\
& \rho A w_{t t}+E I w_{x x x x}+c w_{t}= \delta(x-v t) P(t) \\
&+E A \frac{\partial}{\partial x}\left(e w_{x}\right) .
\end{aligned}
$$

Where

$$
e=u_{x}-u_{x}^{2}+\frac{1}{2} w_{x}^{2}
$$

In these equations, $E$ is Young's modulus of the beam, $\rho$ is the beam density, $A$ is the area and $I$ is moment of inertia of the beam cross-section. $w(t)$ is the vertical deflection of the beam, while $u$ is the axial displacement, $w$ and $u$ depend on the spatial co-ordinate $x$ and the time $t . \delta$ is Dirac delta function and is defined as

$$
\delta(x)=\left\{\begin{array}{l}
1, x=0 \\
0, \text { others }
\end{array}\right.
$$

Assuming that the longitudinal inertial terms $u_{t t}$ and $u_{x}^{2}$ are negligible (case of beam with small radius of gyration), equation (1) and (2) can be reduced to

$$
\begin{aligned}
& \rho A w_{t t}+E I w_{x x x x}+c w_{t}-\frac{3 E A}{2} \frac{\partial^{2} w}{\partial x^{2}}\left(\frac{\partial w}{\partial x}\right)^{2} \\
& =\delta(x-v t) P(t) .
\end{aligned}
$$

If the inertia force of the vehicle is not considered, in other word, the axle force is considered as static weight of the vehicle, $P(t)$ is a constant value. If the vehicle is modeled as Fig. 2, $P(t)$ should be computed as follows.

$$
\begin{gathered}
P(t)=\left(m_{1}+m_{2}\right) g-m_{t} \frac{\partial^{2} y_{t}(t)}{\partial t^{2}}-m_{b} \frac{\partial^{2} y_{b}(t)}{\partial t^{2}} \\
m_{b} \frac{d^{2} y_{b}(t)}{d t^{2}}+c_{b}\left(\frac{d y_{b}}{d t}-\frac{d y_{t}}{d t}\right)+k_{b}\left(y_{b}-y_{t}\right)=0 \\
m_{t} \frac{d^{2} y_{t}(t)}{d t^{2}}+m_{b} \frac{d^{2} y_{b}(t)}{d t^{2}}+c_{a}\left(\frac{d y_{t}}{d t}+\frac{d y_{w}}{d t}\right) \\
+k_{a}\left[y_{t}+w(v t, t)\right]=0 .
\end{gathered}
$$

It is convenient to assume an expansion for the deflection $w$ in terms of the combination of the linear free-oscillation modes, which are those of a hinged-hinged beam in this case. Thus, an expansion is assumed for $w$ in the form

$$
w(x, t)=\sum_{j=1}^{N} Y_{j}(t) \varphi_{j}(x),
$$

where $\varphi_{j}(x)=\sin \frac{j \pi x}{L}$ is the natural mode of a hingedhinged beam.

Substituting Eq. (6) into Eq. (5), multiplying by $\sin \frac{j \pi x}{L}$ and integrating over the interval $[0, \mathrm{~L}]$ leads to the mode equations

$$
\begin{aligned}
& \ddot{Y}_{j}(t)+2 \xi \omega_{j} \dot{Y}_{j}(t)+\omega_{j}^{2} Y_{j}(t)= \\
& \frac{2 P}{m L} \sin j \omega t+\frac{j^{2} \pi^{4} E}{\rho L^{4}} Y_{j} \sum_{k=1}^{\infty} Y_{k}^{2} k^{2} \quad(j=1,2,3, \cdots),
\end{aligned}
$$

where $Y_{j}(t)=(2 / L) \int_{0}^{L} w(x, t) \sin (j \pi x / L) d x$ is the beam displacement of the $j$ th mode, $\xi$ is the damping ratio, $\omega_{j}=\left(\frac{j \pi}{L}\right)^{2} \sqrt{\frac{E I}{m}}$ is the natural angular frequency of the $j$ th mode and $\omega=\frac{\pi v}{L}$.

Since the high order modes of motion contribute little to bending displacement and the nonlinear characters, only the basic mode is considered. Then the equation of motion can be written as

$$
\begin{aligned}
& \ddot{Y}_{1}(t)+\omega_{1}^{2} Y_{1}(t)+2 \xi_{1} \omega_{1} \dot{Y}_{1}(t)+\alpha Y_{1}^{3}(t)= \\
& \frac{2}{m L} P(t) \sin \frac{\pi v t}{L},
\end{aligned}
$$

where $\alpha=\frac{3}{8 r^{2}} \omega_{1}^{2}, r^{2}=\frac{I}{A}$.

\section{Numerical solution and resonance analysis}

For the numerical simulation, the following data are used. For the bridge, the cross-section of the beam is assumed 
as square, and the values of the parameters is assumed as follows, area $A=0.64 \mathrm{~m}^{2}$, section inertia $I=0.0341 \mathrm{~m}^{4}$, span length $l=40 \mathrm{~m}$. For the material, Young's modulus $E=3.0 e 7 \mathrm{kN} / \mathrm{m}^{2}$, density $\rho=2.5 \mathrm{t} / \mathrm{m}^{3}$. For the vehicles, $m_{t}=364 \mathrm{~kg}, m_{b}=1776 \mathrm{~kg}, k_{a}=121 \mathrm{kN} / \mathrm{m}, k_{b}=$ $195.47 \mathrm{kN} / \mathrm{m}, c_{a}=5950 \mathrm{~kg} / \mathrm{s}, c_{b}=3820 \mathrm{~kg} / \mathrm{s}$.

Considering the case only one vehicle pass through the bridge. Using Runge-Kutta method, the computer program is compiled based on software MATLAB. The displacement data of the mid-span is showed in Fig. 3.

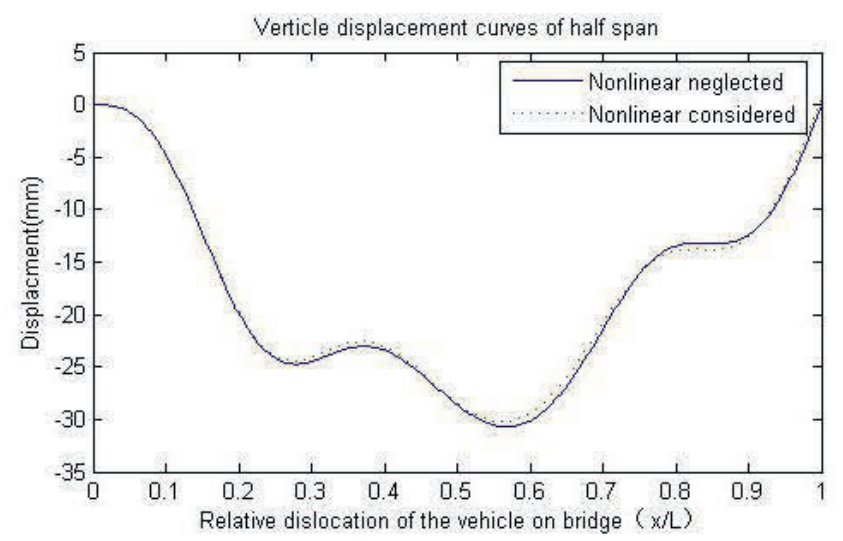

Figure 3. Vertical displacement curves of mid-span.

In Fig. 3, the result of nonlinear case is compared with the case nonlinear not be considered. From Fig. 3, in fact, nonlinear action of the bridge is not obvious when the bridge is forced only one moving vehicle. It has be studied enough in literature [11] about the case one vehicle pass though bridge. Although different vehicle models be used, the conclusion that nonlinear items may lead to the response decreasing is coincident.

When the bridge is subjected with train, the resonance phenomenon should be considered for the periodicity of the train force. In the study [14], the critical velocity of the train is given as

$$
v_{c}=\frac{L_{d} \omega_{0}}{\pi} .
$$

In the above formula, $L_{d}$ is the axle distance, $\omega_{0}$ is the natural frequency of the bridge, $v_{c}$ denotes the velocity value which may lead to the bridge's resonance.

When the nonlinear character can not be ignored, the vehicle-bridge coupling system is the nonlinear dynamical system. For the third power item exist in the nonlinear system, 1/3 subharmonic resonance and 3 times superharmonic resonance may be produced with the period excitation. This coupling system is so complex that it cannot be solved using analysis method. So the numerical method is applied in this paper. In the example, the train is simulated as a series of quarter vehicle model.

It is assumed that the vehicle pass through the bridge with the velocity $v=10 \mathrm{~m} / \mathrm{s}$, which is close to $\omega_{0} / 3$. The

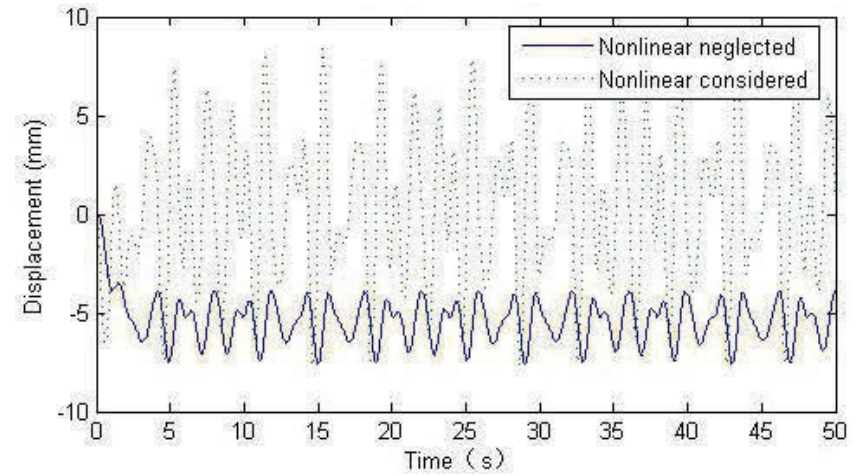

Figure 4. Displacement of mid-span of the bridge under series moving spring-mass system.

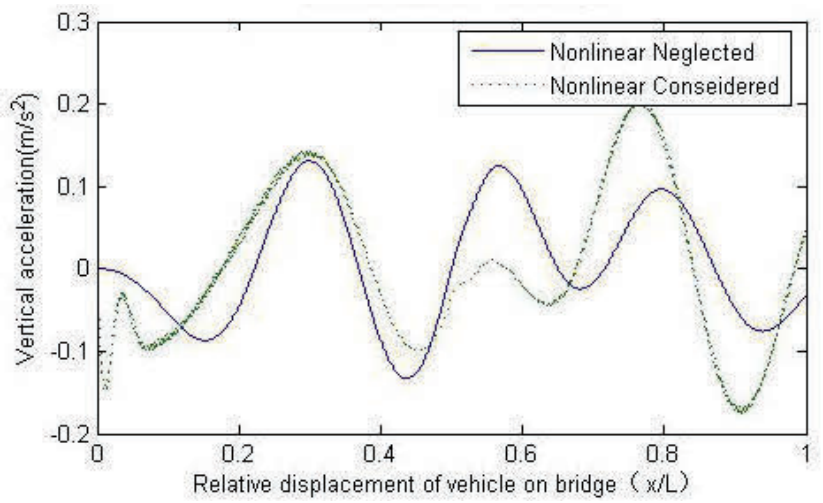

Figure 5. Vertical accelerations of the vehicle passing bridge.

number of train segments is forty. Using the numerical method, the numerical solution of the coupling system is obtained, and the displacement of the mid-span is shown in fig.4. From Fig. 4, the conclusions follow as: (1) although the displacement of the bridge has little changed, the vibration amplitude of the bridge raise obviously when the geometry nonlinear is considered. (2) time history curve shows the harmonic components are ignored.

In the case $1 / 3$ subharmonic resonance, nonlinear effect to the vehicle's vertical acceleration variously. The vertical accelerations of one axle is shown in Fig. 5. The vertical acceleration of vehicle is not reduced but increase when the axle pass through the bridge in region $[\mathrm{L} / 2, \mathrm{~L}]$.

In order to observe harmonic components conveniently, the axle loads are assumed as a series of uniform space constant force. In other words, the vertical inertial force of the train is ignored. The time history curves of the bridge response are shown in Fig. 6.

From Fig. 6, the conclusions can be described as follows: (1) Ignoring the vertical inertial force of the train and the nonlinear of the bridge, the response of the bridge is only a simple harmonic forced vibration. (2) Ignoring the vertical inertial force of the train but not ignoring the nonlinear 


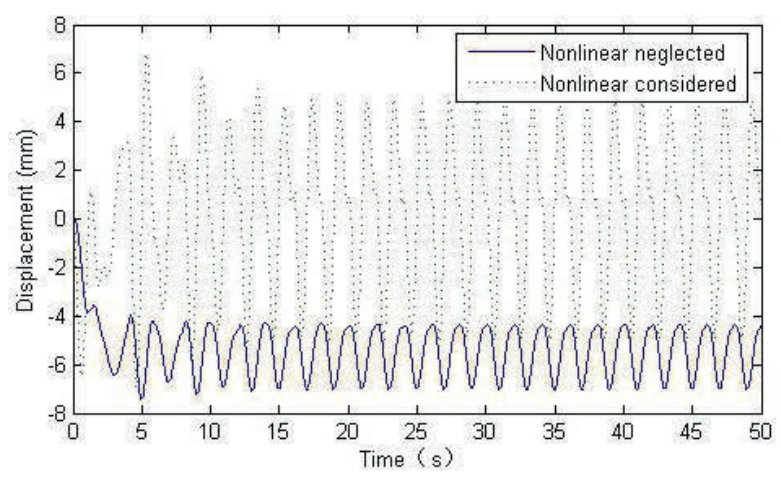

Figure 6. Displacement of mid-span of the bridge under series moving constant force.

of the bridge, the response of the bridge has subharmonic components. In other words, subharmonic resonance exist in the coupling system.

\section{Conclusion}

In this paper, the dynamical response of the vehicle-bridge coupling system is studied with the geometry nonlinear of the bridge being considered. Two case, single vehicle and series of moving axles are discussed. Some conclusions are obtained.

(i) When the bridge is under with only one moving vehicle, the effect of the geometry nonlinear of the bridge to the system response is small.

(ii) When the bridge is under with the series moving axles, nonlinear items of the coupling system affect to the dynamical response of bridge are obviously that it maybe not be ignored in some cases.

(iii) When the moving velocity of the moving axles near the critical value, subharmonic resonance phenomenon is occurred in this coupling system, which is the particular characters of the nonlinear system.

\section{Acknowledgment}

This work is supported by the National Science Foundation of China (Grant Nos: 51108047, 11272270, 11102028) and the Fujian Province National Science Foundation (Grant No-2011J01024).

\section{References}

[1] E.C. Ting, M. Yener. Vehicle structure interactions in bridge dynamics. Shock Vibration Digest. 1983, 15:3-9

[2] D.G. Duffy. The response of an infinite railroad track to a moving vibrating mass. Transactions of the American Society of Mechanical Engineers, Journal of Applied Mechanics. 1990, 57:66-73

[3] F. T. Wang, Vehicle Dynamics, China Railway Press, Beijing, 1994

[4] Y.K. Cheung, F.T.K. Au, D.Y. Zheng, Y.S.Cheng. Vibration of multi-span bridges under moving vehicles and trains by using modified beam vibration functions, Journal of Sound and Vibration, 1999, 228:611-628

[5] J. W. Kwark, E.S. Choi, Y.J. Kim, S.I. Kim. Dynamic behaviour of two-span continuous concrete bridges under moving high-speed train, Computers\&Structure, 2004, 82: 463-474

[6] Q.L. Zhang, Q. Vrouwenvelder, J. Wardenier. Numerical simulation of train-bridge interactive dynamics. Journal of Computers and Stuctures, 2001, 79: 1059-1075

[7] Y.L. Xu, N. Zhang, H. Xia. Vibration of coupled train and cable stayed bridge system in cross wind. Engineering Structures, 2004, 26:1389-1406

[8] Y.B. Yang, J.D. Yau, L.C. Hsu. Vibration of simple beams due to trains moving at high speed. Journal of Engineering Structures, 1997, 19:936-944

[9] N. Zhang, X. He, W.W. Guo. Vehicle-bridge interaction analysis under high-speed trains. Journal of Sound and Vibration, 2008, 309:407-425

[10] H. Xia, Y.L. Xu, T.H.T. Chan. Dynamic interaction of long suspension bridges with running trains. Journal of Sound and Vibration, 2000, 237(2):263-280

[11] K. Liu, G.De Roeck, G. Lombaert. The effect of dynamic train-bridge interaction on the bridge response during a train passage. Journal of Sound and Vibration, 2009,325:240-251

[12] J.F. Wang, C.C. Lin, B.L. Chen. Vibration suppression for high-speed railway bridges using tuned mass dampers. International Journal of Solid and Structures, 2003,40:465491

[13] Y.B. Yang, C.L. Lin, L.D.Yau, D.W.Chang. Mechanism of resonance and cancellation for train-induced vibrations on bridges with elastic bearings. Journal of Sound and Vibration, 2004,269:345-360

[14] Farhad S. Samani, Francesco Pellicano. Vibration reduction on beams subjected to moving loads using linear and nonlinear dynamic absorbers. Journal of Sound and Vibration, 2009,325:742-754

[15] C.Z. Qian, J.S. Tang. A time delay control for a nonlinear dynamic beam with moving load. Journal of Sound and Vibration, 2008, 309:1-8

[16] A.H. Nayfeh, D.T.Mook. Nonlinear oscillations. Wiely, New York, 1979

[17] A.H. Nayfeh. Introduction to perturbation techniques, Wiley, New York, 1981 